\title{
Classical and Variant Avian Infectious Bronchitis Virus Strains ${ }^{1}$
}

Gary D. Butcher, David P. Shapiro, Richard D. Miles, and Jacqueline P. Jacob²

Infectious bronchitis is an acute and highly contagious respiratory disease of chickens. The disease is characterized by respiratory signs including gasping, coughing, sneezing, tracheal rales, and nasal discharge. In young chickens, severe respiratory distress may occur; while in layers, respiratory distress, decrease in egg production, and loss of internal and shell quality of eggs are reported. Some strains of the virus cause severe kidney damage and may be associated with high mortality.

\section{Occurence}

Infectious bronchitis has been reported as a disease in chickens only. All ages of chickens are susceptible to infection, however, clinical disease severity varies. Infectious bronchitis is considered to be worldwide in distribution. The incidence is not constant throughout the year, being reported more often during the cooler months.

\section{History}

The disease was first described in 1931 in a flock of young chickens in the USA. Since that time, the disease has been identified in broilers, layers and breeder chickens throughout the world. Vaccines, to help reduce losses in chickens, were first used in the 1950s.

\section{Etiology}

Infectious bronchitis is caused by a coronavirus. It is an enveloped, single stranded RNA virus. Three virus-specific proteins have been identified; the spike (S) glycoprotein, the membrane or matrix $(\mathrm{M})$ glycoprotein, and the nucleocapsid $(\mathrm{N})$ protein (Figure $1)$.

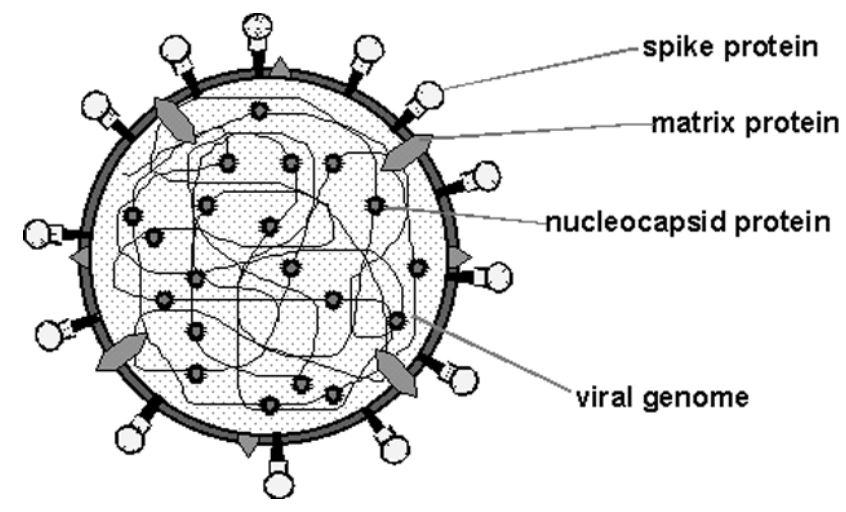

Figure 1. Infectious bronchitis virus proteins.

The crucial spike glycoprotein is comprised of two glycopolypeptides (S1 and S2). These spikes or peplomers can be seen projecting through the envelope on electron micrographs giving the virus its characteristic 'corona' (Figure 2). Hemaglutination inhibition and most serum nutralization antibodies are directed against this $\mathrm{S} 1$ portion. The unique amino

1. This document is FACT SHEET PS-40, one of a series of the Dairy and Poultry Sciences Department, Florida Cooperative Extension Service, Institute of Food and Agricultural Sciences, University of Florida. First published June 1998. Please visit the EDIS Website at http://edis.ifas.ufl.edu.

2. Gary D. Butcher, extension veterinarian, College of Veterinary Medicine; David P. Shapiro, Hoechst Roussel Vet, Knoxville, Tennessee 37921; Richard D. Miles, professor, and Jacqueline P. Jacob, poultry extension coordinator, Dairy and Poultry Sciences Department, Cooperative Extension Service, Institute of Food and Agricultural Sciences, University of Florida, Gainesville, 32611. educational information and other services only to individuals and institutions that function without regard to race, color, sex, age, handicap, or national origin. For information on obtaining other extension publications, contact your county Cooperative Extension Service office. Florida Cooperative Extension Service / Institute of Food and Agricultural Sciences / University of Florida / Christine Taylor Waddill, Dean 
acid sequences, called epitopes, on this glycoprotein determine the serotype.

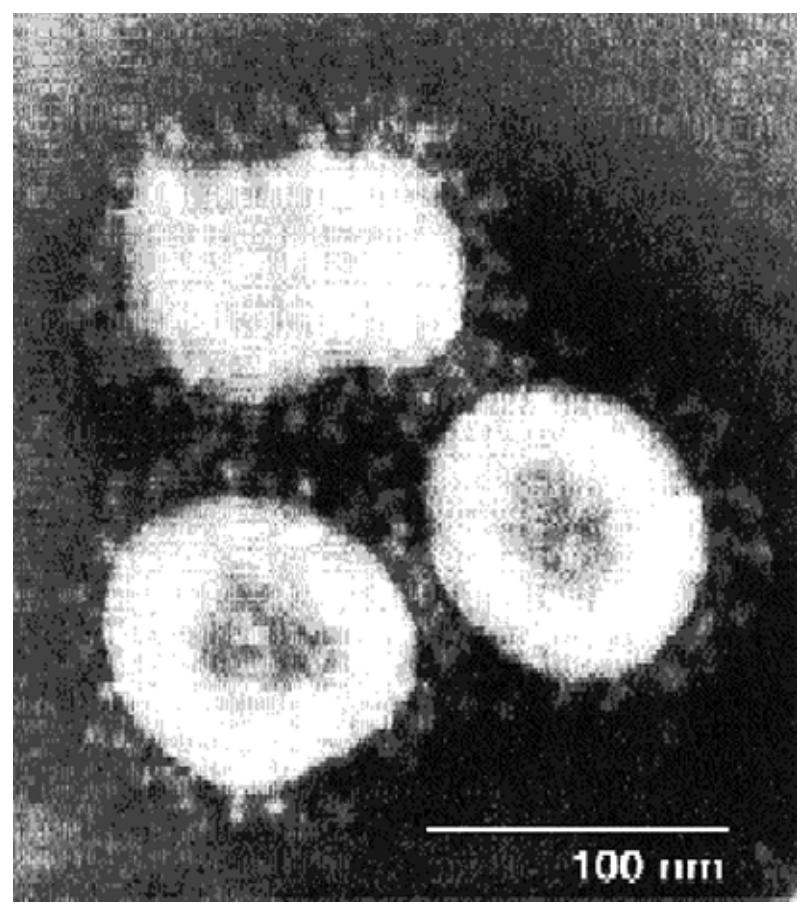

Figure 2. Electron Micrograph illustrating the appearance of a coronavirus.

The virus is fairly labile (fragile), being easily destroyed by disinfectants, sunlight, heat and other environmental factors. Infectious bronchitis virus has the ability to mutate or change its genetic makeup readily. As a result, numerous serotypes have been identified and have complicated efforts at control through vaccination. Three common serotypes in North America are the Massachusetts, Connecticut, and Arkansas 99 infectious bronchitis viruses. In Europe various "Holland variants," usually designated using numbers (D-274, D-212), are recognized.

Several strains of infectious bronchitis virus have a strong affinity for the kidney (nephropathogenic strains). These strains may cause severe renal damage. This affinity for kidney tissue may have resulted from mutation as a result of selection pressure following widespread use of the modified live infectious bronchitis vaccines. That is, after prolonged use of live infectious bronchitis vaccines, which provided protection against infectious bronchitis virus infection in respiratory tissues, new tissues where little protection was present were infected as a result of viral mutation. These viruses have become more prevalent in recent years.

\section{Transmission}

Infectious bronchitis virus is spread by the respiratory route in droplets expelled during coughing or sneezing by infected chickens. Spread of the disease through a flock is very rapid. Transmission from farm to farm is related to movement of contaminated people, equipment, and vehicles. Following infection, chickens may remain carriers and shed the virus for several weeks. The virus is not transmitted through eggs.

\section{Clinical Signs}

\section{Chicks}

Clinical signs include coughing, sneezing, rales, nasal discharge, and frothy exudate in the eyes. Affected chicks appear depressed and will tend to huddle near a heat source. In an affected flock, all birds will typically develop clinical signs within 36 to 48 hours. Clinical disease will normally last for 7 days. Mortality is usually very low, unless complicated by other factors such as M. gallisepticum, immunosuppression, poor air quality, etc.

\section{Chickens}

Clinical signs such as coughing, sneezing and rales may be observed. A drop in egg production of 5 to 10 percent lasting for 10 to 14 days is commonly reported. However, if complicating factors are present, production drops may be as high as 50 percent. Eggs produced following infection may have thin shells, irregular shells, and thin, watery albumen. Loss of pigment in brown-shelled eggs is common. In severe complicated cases, chickens may develop airsacculitis. Chickens that experienced a severe vaccination reaction following chick vaccination or field infection during the first 2 weeks of life may have permanent damage in the oviduct, resulting in hens with poor egg production.

In recent years, nephropathogenic strains have become more common in laying flocks. These strains may cause an elevated mortality during the infection 
or long after as a result of kidney damage that progresses to urolithiasis.

\section{Lesions}

Lesions associated with infectious bronchitis include a mild to moderate inflammation of the upper respiratory tract. If complicating factors are present, airsacculitis and increased mortality may be noted, especially in younger chickens. Kidney damage may be significant following infection with nephropathogenic strains. Kidneys of affected chickens will be pale and swollen. Urate deposits may be observed in the kidney tissue and in the ureters, which may be occluded. Laying chickens may have yolk material in the body cavity and developing yolks in the ovary may be flaccid. Infection of very young chicks may result in the development of cystic oviducts.

\section{Diagnosis}

To determine if a response to infectious bronchitis virus has occurred in a suspect flock, serologic testing is performed by comparing two sets of serum samples. One collected at onset of clinical disease and the second at $31 / 2$ to 4 weeks later. Serologic procedures commonly used include ELISA, virus neutralization, and hemaglutination inhibition.

Confirmation of infectious bronchitis requires isolation and identification of the virus. Typically this is done in specific pathogen-free chicken embryos at 9 to 11 days of incubation by the allantoic sac route of inoculation. Tissues collected from diseased chickens for virus isolation attempts include trachea, lungs, airsacs, kidney, and cecal tonsils. If samples are collected more than 1 week after infection, cecal tonsils and kidneys are the preferred sites for recovery of infectious bronchitis virus.

Virus typing has traditionally been performed by neutralization using selected infectious bronchitis antisera. More recently, polymerase chain reaction (PCR) and restriction fragment length polymorphism (RFLP) have been used to differentiate infectious bronchitis virus serotypes. Lesions in embryos are helpful in diagnosing infectious bronchitis. Affected embryos examined at 7 days after inoculation are stunted, have clubbed down, an excess of urates in the kidneys, and the amnion and allantois membranes are thickened and closely invest the embryo. These embryos will not hatch. The infectious bronchitis field virus may need to be repeatedly passed in embryos to adapt the virus before these typical lesions are recognized. On initial passages, the infectious bronchitis field virus will grow poorly and only mild lesions will be observed. Following serial passage and adaptation, the virus will replicate more efficiently and be able to cause more severe embryonic lesions.

\section{Control}

Prevention of infectious bronchitis is best achieved through an effective biosecurity program. As a second line of defense, chickens in infectious bronchitis problem areas should be vaccinated with modified live vaccines to provide protection. The multiplicity of serotypes identified in the field presents a challenge in designing an effective vaccination program. To be successful in protecting chickens against infectious bronchitis, it is essential to identify the prevalent serotypes in the region and to determine the cross-protective potential of available vaccines. In North America, the common serotypes used in most vaccination programs are the Massachusetts, Connecticut, and Arkansas serotypes. These serotypes are availabe in both modified live vaccines and inactivated water-in-oil emulsions. Regionally important serotypes (e.g., California strains) may be included in inactivated vaccines. Polyvalent vaccines, which contain multiple strains, are also available. Control of other respiratory diseases (e.g., Newcastle, Mycoplasma gallisepticum) and strongly immunosuppressive diseases (e.g., Infectious bursal disease, Marek's disease) must not be forgotten.

\section{Vaccine Selection}

Infectious bronchitis vaccination programs in broilers involve the use of modified live vaccines. Vaccination of layers has historically involved administering a series of live vaccines and progressively increasing the aggressiveness of the route of vaccination (i.e., start with water administration and progress to fine particle spray) and strain of vaccine (highly attenuated to less attenuated).

In breeders, a similar program is often followed. However, prior to onset of egg production, an 
inactivated vaccine is also administered to stimulate antibody production. Inactivated vaccines stimulate higher levels of circulating antibodies than live vaccines and would be of value in a breeder program where maternal antibody protection is needed. However, modified live vaccines provide better stimulation of the cell mediated system ( $\mathrm{T}$ cell) and elicit a superior local antibody (IgA) response as a result of local mucosal infection and thus would be of more value than inactivated vaccines in protecting commercial layers.

With dozens of infectious bronchitis virus strains having been identified around the world, choosing appropriate strains for vaccination may seem a daunting task. The immune response to one strain, however, often shows a significant degree of cross-protection to heterologous challenge. Crossprotection has been demonstrated especially for the live-type vaccines. If the prevalent strains for a region have been identified, it is often possible to design a program using commercially available vaccine strains (Table 1).

Although no reasonable combination of infectious bronchitis vaccine strains provides full protection against all heterologous challenges, there are combinations which are broad in coverage. Once the prevalent serotypes in an area have been identified, modified live vaccines containing carefully chosen strains can be used to immunize broilers, layers and breeders. Additionally, polyvalent inactivated vaccines can be administered to breeders at point-of-lay.
It has been demonstrated that "classical" strains of infectious bronchitis virus can act at least as partial primers for subsequent administration of an inactivated infectious bronchitis vaccine containing variant and standard strains. Inactivated infectious bronchitis vaccines do not stimulate local and cell-mediated immunity as effectively as modified live virus infectious bronchitis vaccines, however, they can provide a degree of immunity against variant strains without the risk of introducing new strains of infectious bronchitis into a poultry operation. Imprudent overuse of live nfectious bronchitis vaccines results in the vaccines being the problem rather than part of the solution.

While deciding which strains to utilize in an infectious bronchitis vaccination program, the basics must not be ignored. Good vaccination practices are especially important when administering live infectious bronchitis vaccines. It is a relatively fragile virus and can easily be inactivated if proper vaccination procedures (e.g., protection from sunlight, removal of sanitizers from water used for mixing/administration, use of skim milk stabilizer, etc.) are not followed. 
Table 1. Percent cross-protection afforded Specific-pathogen-free White Leghorn chickens vaccinated by eyedrop at 2 to 3 weeks of age with live infectious bronchitis virus (IBV) vaccines and challenged 4 weeks later with homologous and heterologous reference strains and variant field isolates. (The numbers indicate percent protection - the percentage of chickens not yielding virus from tracheal swabbings collected 5 days after challenge-virus inoculation. "Mean \%=" represents percent protection of an IBV vaccine against reference strain or layer variant strain challenge.)

\begin{tabular}{|c|c|c|c|c|c|}
\hline \multirow{2}{*}{ Challenge Virus } & \multicolumn{5}{|c|}{ VACCINE } \\
\hline & $\begin{array}{l}\text { Mass } \\
\text { (Holland) }\end{array}$ & $\begin{array}{l}\text { Mass (L-1) } \\
+ \text { Conn }\end{array}$ & $\begin{array}{l}\text { Mass (Holland) } \\
+ \text { Ark }\end{array}$ & $\begin{array}{l}\text { Mass (L-1) } \\
+ \text { Ark }\end{array}$ & $\begin{array}{l}\text { Mass } \\
\text { (Connaught) + Ark }\end{array}$ \\
\hline Mass 41 & $84 \%$ & $93 \%$ & $87 \%$ & $86 \%$ & $100 \%$ \\
\hline Ark DPI & 47 & 27 & 87 & 100 & 93 \\
\hline Conn & 57 & 100 & 100 & 87 & 100 \\
\hline JMK & 80 & 86 & 73 & 93 & 93 \\
\hline Holte & 70 & 33 & 79 & 40 & 93 \\
\hline Florida & 77 & 80 & 78 & 60 & 80 \\
\hline mean $\%=$ & 69 & 70 & 84 & 78 & 93 \\
\hline \multicolumn{6}{|l|}{ Layer variants } \\
\hline $46 \mathrm{C}$ & $27 \%$ & $33 \%$ & $47 \%$ & $73 \%$ & $40 \%$ \\
\hline 16VT & 47 & 20 & 73 & 60 & 67 \\
\hline $33 \mathrm{VT}$ & 60 & 13 & 80 & 87 & 60 \\
\hline 3330 & 47 & 13 & 87 & 53 & 53 \\
\hline mean $\%=$ & 45 & 20 & 71 & 68 & 55 \\
\hline \multicolumn{6}{|l|}{ Broiler variant } \\
\hline 06 & $93 \%$ & $100 \%$ & $80 \%$ & $87 \%$ & $80 \%$ \\
\hline
\end{tabular}

(adapted from Gelb et al., Variant Serotypes of Infectious Bronchitis Virus Isolated from Commercial and Broiler Chickens. Avian Diseases 35:82-87, 1991.) 